\title{
Measurement of electron wave functions and confining potentials via photoemission
}

\author{
A. Mugarza, ${ }^{1,2}$ J. E. Ortega, ${ }^{1,2}$ F. J. Himpsel, ${ }^{3}$ and F. J. García de Abajo ${ }^{1,4, *}$ \\ ${ }^{1}$ Donostia International Physics Center (DIPC), Aptdo. 1072, 20080 San Sebastián, Spain \\ ${ }^{2}$ Departamento de Física Aplicada I, Universidad del País Vasco, Plaza Oñati 2, 20018 San Sebastián, Spain \\ ${ }^{3}$ Department of Physics, University of Wisconsin, Madison, Wisconsin 53706 \\ ${ }^{4}$ Centro Mixto de Materiales CSIC/UPV, Paseo Manuel Lardizabal 3, E-20018 San Sebastian, Spain
}

(Received 12 December 2002; published 28 February 2003)

\begin{abstract}
Wave functions and electron potentials of laterally confined surface states are determined experimentally by means of photoemission from stepped $\mathrm{Au}(111)$ surfaces. The confined nature of the wave function makes it possible to use an iterative formalism borrowed from x-ray diffraction where real-space wave functions are retrieved from the absolute values of their Fourier transforms. The latter are measured by angle-resolved photoemission. The effective confining potential is then obtained by introducing the wave functions into Schrödinger's equation.
\end{abstract}

DOI: 10.1103/PhysRevB.67.081404

PACS number(s): 79.60.Bm, 68.35.Bs, 73.21.-b

The electron wave functions and potentials in lowdimensional systems are of primary importance for tailoring electronic properties of nanostructures. The electron energy levels and the probability density are the physical observables. These can be obtained in real space from local conductance maps via scanning tunneling microscopy/ spectroscopy (STM/STS), ${ }^{1,2}$ or in reciprocal space by angleresolved photoemission. ${ }^{3,4}$ The latter has been used to study thin films ${ }^{3}$ and two-dimensional (2D) arrays of nanoobjects, ${ }^{4}$ whereas STM/STS resolves individual nanostructures on a surface.

The question arises whether it is possible to directly derive wave functions and/or electron potentials from experimental data. The standard procedure consists of assuming a model potential, whose parameters are obtained by fitting the experiment. For instance, in core-level photoelectron diffraction, muffin-tin potentials are assumed for retrieving atomic positions that fit the experiment. ${ }^{5,6}$ The electron density of surface states confined between two steps has been measured by STM (Refs. 1 and 2) and has been modeled by semitransparent mirrors located at the step edges. ${ }^{2}$ It has been argued that the position of the mirror has to be moved slightly away from the steps to account for electron spillover. ${ }^{7}$

We introduce a direct method to determine the wave function and the effective electron potential from angle-resolved photoemission data, without any a priori knowledge about their nature. All the necessary information is contained in the momentum distribution of the photoemission intensity, as long as the wave function is confined to finite area (a terrace in our case). This allows us to unambiguously determine the potential and wave functions of one-dimensional quantumwell states on stepped $\mathrm{Au}(111)$. The real-space wave functions are derived from the Fourier transform of their momentum-space representations, the square of which is proportional to the photoelectron intensity under the conditions discussed below. However, the phase in momentum space is not measured and this leads to the well-known phase problem in optics, shared with many other techniques, such as X-ray and electron diffraction. 8,9 Various iteration methods have been devised to retrieve real-space objects from the modulus of their momentum-space representation, in particu- lar, the oversampling method. ${ }^{10}$ These methods are valid for a confined wave function because the phase is obtained by repeatedly diminishing the amplitude of the wave function outside the confinement region. The one-electron potential can be obtained after dividing the Schrödinger equation by the wave function.

As a test system we use vicinal noble-metal surfaces char-
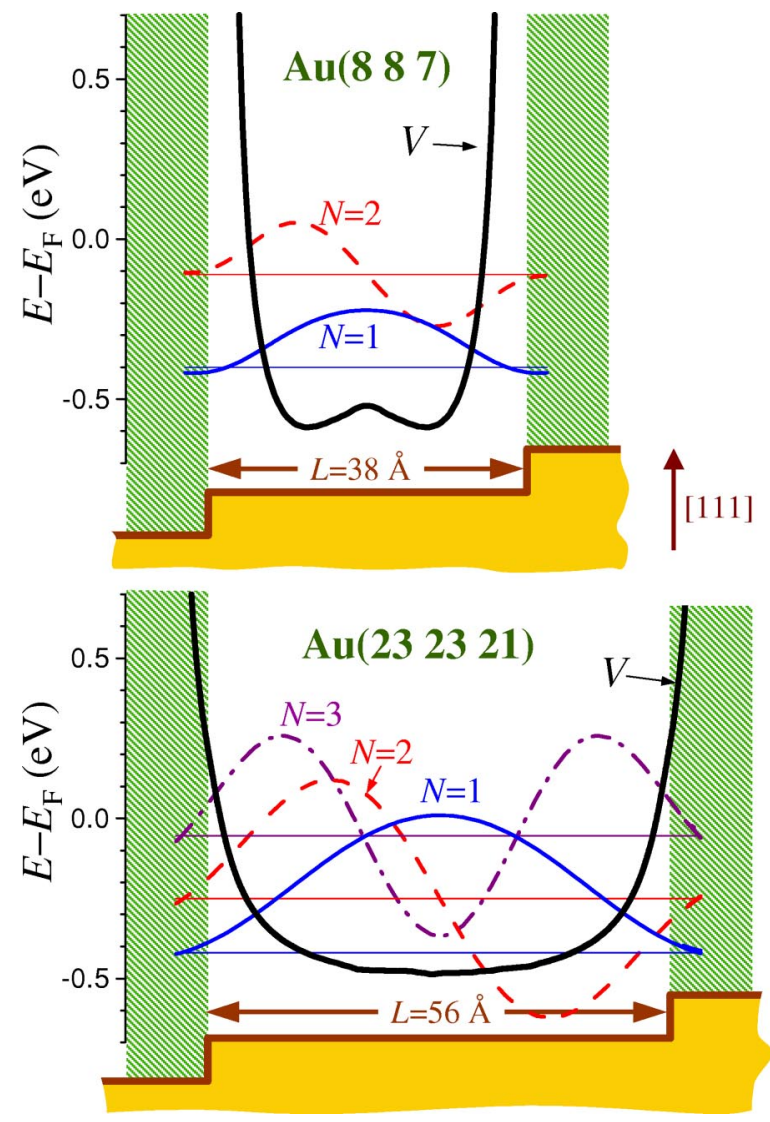

FIG. 1. Vicinal $\mathrm{Au}(111)$ surfaces with quantum-well states confined by step edges. The energy levels are directly measured by angle-resolved photoemission. The confining potential $V$ and the wave functions of states $N=1,2,3$ are obtained from the momentum distribution of the photoemission intensity. 
(a) $\mathrm{Au}(887)$

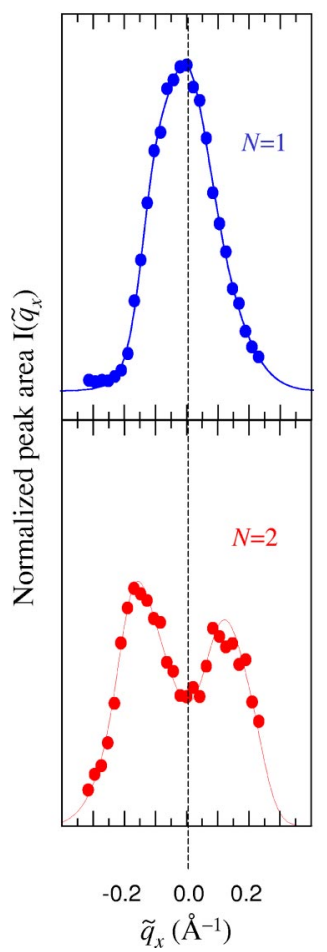

(b) $\operatorname{Au}(232321)$

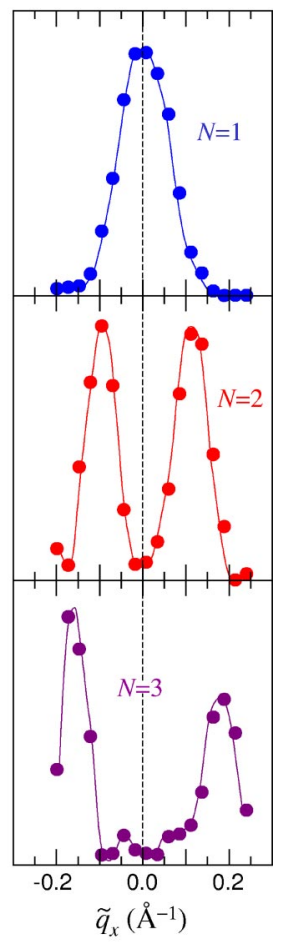

FIG. 2. Angular distribution of the photoemission intensity from one-dimensional quantum-well states at vicinal (a) $\mathrm{Au}(887)$ and (b) $\operatorname{Au}(23,23,21)$ surfaces. The lines are smooth (spline) fits to the data points and are used as input intensity functions in Eq. (3) (see the text).

acterized by equally spaced, linear step arrays and onedimensional surface states confined within individual terraces of nominal size $L$. This is the case of $\mathrm{Au}(111)$ vicinal surfaces with relatively wide terraces, such as $\mathrm{Au}(887)$ ( $L$ $=38 \AA)$ and $\mathrm{Au}(23,23,21)(L=56 \AA)$ shown in Fig. 1 (Refs. 4 and 11). $\mathrm{Au}(887)$ is characterized by two flat quantum levels $(N=1-2)$ below the Fermi energy, whereas the wider terraces in $\operatorname{Au}(23,23,21)$ allow for three occupied quantumwell levels $(N=1-3)$. Data points in Figs. 2(a) and 2(b) show the angular photoemission intensity scan for each quantum level as obtained from the areas of the peaks in individual photoemission spectra. ${ }^{4,11}$ Data for $\mathrm{Au}(887)$ were taken at the SU8 beam line at the synchrotron LURE in Paris (France) using a VSW electron analyzer, whereas $\mathrm{Au}(23,23,21)$ data were taken at the PGM beam line in the Synchrotron Radiation Center (SRC) of the UW-Madison, using a hemispherical Scienta SES200 spectrometer. The experiment details have been published elsewhere. ${ }^{4,11}$ The intensity is properly normalized to account for the photon flux and the transmission function in the Scienta analyzer. The lines represent a smooth (spline) fit to data points that go to zero, away from the displayed wave-vector region in Fig. 2, where the photoemission spectra are completely featureless.

The photoemission intensity in reciprocal space $\mathbf{q}$ can be understood in the framework of the standard photoemission theory, assuming a one-electron description. We start with the photoemission intensity, which is proportional to the matrix element

$$
I_{N}(\mathbf{q} \|) \propto\left|\left\langle\mathbf{q}\left|e^{i \mathbf{k} \cdot \mathbf{r}} \mathbf{A} \cdot \nabla\right| \Psi_{N}\right\rangle\right|^{2} .
$$

$\Psi_{N}$ is the initial surface-state wave function, $|\mathbf{q}\rangle$ is the final electron state of momentum $\mathbf{q}=\left(\mathbf{q}_{\|}, q_{z}\right), \mathbf{q}_{\|}$is the momentum component parallel to the surface, $\mathbf{A}$ is the light polarization vector, $\mathbf{k}$ is the momentum of the photon, and $N$ refers to the quantum number of the wave function. To proceed further, it will be assumed that the surface-state wave functions can be factorized into components that are either parallel or perpendicular to the surface, i.e., ${ }^{12}$

$$
\Psi_{N}(\mathbf{r})=\phi_{N}(\mathbf{R}) \varphi(z),
$$

where $\mathbf{R}$ denotes the coordinates along the surface. If, in addition to the surface-state confinement in the perpendicular direction $z$, the electron is also bound to a $2 \mathrm{D}$ region of the surface, the parallel component of the wave function $\phi_{N}(\mathbf{R})$ must adopt a form that depends on the detailed shape of the confining region and on the boundary conditions at its border. Thus, $I_{N}$ can be written as

$$
I_{N}\left(\mathbf{q}_{\|}\right) \propto \quad C\left|\left\langle\mathbf{q}_{\|} \mid \phi_{N}\right\rangle\right|^{2},
$$

where

$$
C=\left|\left\langle q_{z}\left|e^{i k_{z} z} A_{z} \frac{\partial}{\partial z}\right| \varphi\right\rangle\right|^{2}
$$

depends very weakly on $\mathbf{q}_{\|}$, so that it can be absorbed into a normalization factor that we set to 1 . Therefore, there is a direct relation between photoemission intensity $I_{N}\left(\mathbf{q}_{\|}\right)$and the Fourier transform of the wave function $\widetilde{\phi}_{N}(\mathbf{q} \|)$ :

$$
\widetilde{\phi}_{N}\left(\mathbf{q}_{\|}\right)=\left\langle\mathbf{q}_{\|} \mid \phi_{N}\right\rangle=\int d \mathbf{R} e^{-i \mathbf{q} \| \cdot \mathbf{R}} \phi_{N}(\mathbf{R}) .
$$

Thereby, we neglect multiple scattering in the final state, which at the atomic level is partly due to the lateral atomic corrugation, small compared to that in the bulk. Also, the probability of the surface-state electron to propagate elastically towards the bulk region and back to the vacuum is small due to inelastic attenuation. Multiple scattering involving different terraces can also play a role resulting in Fourier components $\mathbf{q}_{\|}+\mathbf{g}_{\|}$with a reciprocal terrace-lattice vector $\mathbf{g}_{\|}$. However, these affect the intensity distribution between different Brillouin zones but not the momentum distribution within one Brillouin zone (compare this to the spot profiles in low-energy electron diffraction, which are not affected by elastic scattering). ${ }^{13}$ This allows one to obtain the wave function in real space $\phi_{N}(\mathbf{R})$ from the intensity in reciprocal space as

$$
\phi_{N}(\mathbf{R})=\int \frac{d \mathbf{q}_{\|}}{(2 \pi)^{2}} e^{i \mathbf{q} \| \cdot \mathbf{R}} \sqrt{I_{N}\left(\mathbf{q}_{\|}\right)} e^{i \delta_{N}(\mathbf{q} \|)} .
$$

However, this equation still contains an unknown phase $\delta_{N}(\mathbf{q} \|)$. We have explored two methods to obtain this phase: (1) an iterative procedure using oversampling ${ }^{8-10}$ and (2) an expansion of the wave function into a Fourier series combined with a least-squares fit. In both cases, the strategy minimizes $|\phi|$ outside the confinement region. 
For the iterative method, we begin with a constant phase $\delta_{N}(\mathbf{q} \|)=0$ and the smooth fitting curves of Fig. 2 as input data to start the iteration with Eq. (3). The resulting $\phi_{N}(\mathbf{R})$ is corrected outside the confining region, and transformed back into $\mathbf{q}_{\|}$space using Eq. (2). The phase of $\widetilde{\phi}_{N}\left(\mathbf{q}_{\|}\right)$is extracted and inserted again into Eq. (3) to start a new iteration. The confinement length is obtained directly from the photoemission data (see below), and the noted correction consists of subtracting from the newly calculated real-space wave function the wave function at the previous step multiplied by a factor 0.1 outside the confinement region. This ensures convergence to a wave function that vanishes outside the confinement region. As a strong test of convergence of this iterative technique, we have used the phase of the $N=2$ state to start the iteration of the $N=1$ state. The first iteration step leads to a wave function that resembles that of the $N=2$ state, a clear indication of the importance of the phase, ${ }^{8}$ and convergence to the true $N=1$ state is achieved after several hundred iterations.

This iterative method leads to results that are in agreement with those derived from an expansion of the wave function into sine functions that vanish at the edges of the confinement region, and therefore, we only present the results derived from the iterative method, which can be easily generalized to more complicated systems. In either case, the length of the region where the wave function takes nonnegligible values is not sensitive to the input value for the length of the confinement region.

The size of the confining region is obtained from the Fourier transform of the experimental intensity distribution, which can be written as the self-convolution of the real-space wave function,

$\int \frac{d \mathbf{q}_{\|}}{(2 \pi)^{2}} e^{i \mathbf{q} \|} \cdot \mathbf{R}_{N}\left(\mathbf{q}_{\|}\right)=\int d \mathbf{R}^{\prime} \phi_{N}\left(\mathbf{R}^{\prime}\right) \phi_{N}^{*}\left(\mathbf{R}^{\prime}-\mathbf{R}\right)$.

This convolution takes nonzero values in an area twice the size of the confining region. For the data of Fig. 2, in particular, one obtains a function which is basically zero outside a region of $\approx 80 \AA$ and $\approx 120 \AA$ in diameter (not shown), in agreement with terrace-width confinement in $\mathrm{Au}(887)$ and $\mathrm{Au}(23,23,21)$, respectively.

The natural normalization for both the two-dimensional wave function $\phi_{N}$ and the measured intensity is provided by Eq. (4) if one sets $\mathbf{R}=0$ :

$$
\int \frac{d \mathbf{q}_{\|}}{(2 \pi)^{2}} I_{N}\left(\mathbf{q}_{\|}\right)=\left\langle\phi_{N} \mid \phi_{N}\right\rangle=1 .
$$

Applying this procedure to the angular scans of Figs. 2(a) and 2(b), we have obtained the surface-state wave functions for quantum-well levels that are shown in Fig. 1. All wave functions are confined to a region whose width matches the terrace width $L$. Thus, the wave functions exhibit a clear terrace confinement that has not been assumed by our reconstruction procedure, but rather it has emerged from the information contained in the photoemission data.

The actual effective potential of the terrace can be retrieved from the Schrödinger equation as

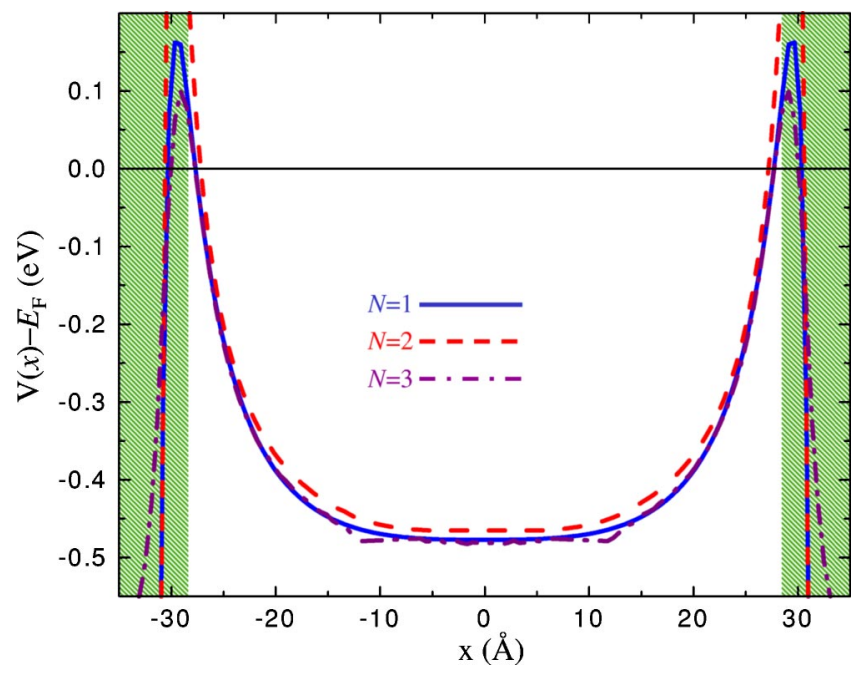

FIG. 3. Confining potentials $V$ obtained from the experimental photoemission intensities of Fig. 2 for the $N=1,2,3$ states in $\mathrm{Au}(23,23,21)$ using the oversampling method.

$$
V(\mathbf{R})-E_{N}=\frac{\hbar^{2}}{2 m^{*} \phi_{N}(\mathbf{R})} \nabla^{2} \phi_{N}(\mathbf{R})
$$

where $m^{*}=0.26 m$ is the effective mass of the electron in the initial state of energy $E_{N}$, taken from the dispersion of the surface state on flat $\mathrm{Au}(111)$. The potential becomes independent of the quantum number $N$ if many-electron effects are absent, as we assumed initially. The effective potential can also change due to a different wave-function penetration inside the bulk, although this effect is expected to be negligible since quantum levels are separated by energies much smaller than the vertical confining barrier (crystal gap).

For $\operatorname{Au}(23,23,21)$, the experimental wave functions of Fig. 1 have been introduced into Eq. (5) and the resulting electron potential has been represented in Fig. 3. In order to compare the potential derived from the quantum states with various $N$, we add on the left-hand side of Eq. (5) the respective experimental values $E_{N}^{\exp }$ shown in Fig. 1 (horizontal bars). An excellent mutual agreement is obtained for the shape of the potential derived independently from each of the three wave functions $N=1-3$, proving the validity of our method. The potential exhibits a smooth central region and sharp boundaries that force electron confinement. It must be stressed that the validity of Eq. (5) is limited to regions where the wave function is not too small, and therefore, the asymptotic limit of the step barrier potential cannot be determined.

The thick lines in Fig. 1 represent the average potential obtained from Eq. (5) for both $\mathrm{Au}(23,23,21)$ and $\mathrm{Au}(887)$ using the wave functions of the different quantum levels in each case, shown in Fig. 1. The width of the potential well in $\mathrm{Au}(887)$ is clearly narrower than the terrace size, as also observed in thin $\mathrm{Pb}$ films. ${ }^{7}$ Near the step, the potential exhibits a steeper increase in $\mathrm{Au}(887)$ than in $\mathrm{Au}(23,23,21)$, which could be explained by a sharper terrace-width distribution in the former case. ${ }^{14}$ 
In summary, we have introduced a simple procedure for directly obtaining wave functions and effective potentials of confined electronic states from the momentum distribution of the photoemission intensity. This procedure, which relies on photoemission theory within the one-electron approach, has been successfully tested in the case of laterally confined surface states on $\mathrm{Au}(111)$ vicinal surfaces. The method is of general applicability for discrete states because they are spatially confined and allow an iterative determination of the phase by forcing $\phi=0$ outside the confinement region. It can be applied to any type of surface nanostructure confined in at least one dimension, such as arrays of quantum wires and quantum dots. While STM provides direct images of the low spatial frequencies, photoemission determines the high spatial frequencies. It would be interesting to pursue the reverse

*Corresponding author.

${ }^{1} \mathrm{Ph}$. Avouris and I.-W. Lyo, Science 264, 942 (1994).

${ }^{2}$ L. Bürgi, O. Jeandupeux, A. Hirstein, H. Brune, and K. Kern, Phys. Rev. Lett. 81, 5370 (1998).

${ }^{3}$ R.K. Kawakami, E. Rotenberg, H.J. Choi, E.J. Escorcia-Aparicio, M.O. Bowen, J.H. Wolfe, E. Arenholz, Z.D. Zhang, N.V. Smith, and Z.Q. Qiu, Nature (London) 398, 132 (1999).

${ }^{4}$ A. Mugarza, A. Mascaraque, V. Pérez-Dieste, V. Repain, S. Rousset, F.J. Garcia de Abajo, and J.E. Ortega, Phys. Rev. Lett. 87, 107601 (2001).

${ }^{5}$ S. Kono, C.S. Fadley, N.F.T. Hall, and Z. Hussain, Phys. Rev. Lett. 41, 117 (1978).

${ }^{6}$ F.J. García de Abajo, M.A. Van Hove, and C.S. Fadley, Phys. Rev. B 63, 075404 (2001).

${ }^{7}$ R. Otero, A.L. Vázquez de Parga, and R. Miranda, Surf. Sci. 447, 143 (2000).

${ }^{8}$ D.K. Saldin, R.J. Harder, V.L. Shneerson, and W. Moritz, J. Phys.: Condens. Matter 13, 10689 (2001).

${ }^{9}$ D.K. Saldin, A. Seubert, and K. Heinz, Phys. Rev. Lett. 88, 115507 (2002) transformation from the real space $|\phi|$ in STM to $\widetilde{\phi}$ in $q$ space using the technique described here. A connection between $|\phi|$ and the $E(q)$ band dispersion has been made already. ${ }^{2,15}$

A.M. and J.E.O. are supported by the Universidad del País Vasco (Grant No. 9/UPV 00057.240-13668/2001) and the Max Planck Research Institute. J.G.A. gratefully acknowledges support by the Basque Departamento de Educación, Universidades e Investigación, the University of the Basque Country UPV/EHU (Contract No. 00206.215-13639/ 2001), and the Spanish Ministerio de Ciencia y Tecnología (Contract No. MAT2001-0946). F.J.H. acknowledges support by the NSF under Grants Nos. DMR-9815416 and DMR0084402 (SRC).

${ }^{10}$ J. Miao, P. Charalambous, J. Kirz, and D. Sayre, Nature (London) 400, 342 (1999); J. Miao, D. Sayre, and H.N. Chapman, J. Opt. Soc. Am. A 15, 1662 (1998).

${ }^{11}$ A. Mugarza, A. Mascaraque, V. Repain, S. Rousset, K.N. Altmann, F.J. Himpsel, Yu. M. Kokoteev, E.V. Chulkov, F.J. García de Abajo, and J.E. Ortega, Phys. Rev. B 66, 245419 (2002).

${ }^{12}$ The approximation of a factorized wave function is reasonable for states that are confined to a single terrace island. It breaks down for a superlattice with strong overlap between adjacent terraces where $\phi$ is delocalized and discrete states become bands.

${ }^{13}$ M. Henzler, Appl. Phys. 9, 11 (1976).

${ }^{14}$ The barrier appears slightly sharper than the terrace width distribution in both surfaces. This could be due to the background removal in line fitting to individual photoemission spectra, which tends to filter out contributions coming from the smallest and the largest terraces [at higher and lower binding energies, respectively (Ref. 11)].

${ }^{15}$ J.I. Pascual, Z. Song, J.J. Jackiw, K. Horn, and H.-P. Rust, Phys. Rev. B 63, 241103 (2001). 